Georgian Mathematical Journal

Volume 14 (2007), Number 4, 627-642

\title{
A MODIFIED QUASI-REVERSIBILITY METHOD FOR A CLASS OF ILL-POSED CAUCHY PROBLEMS
}

\author{
NADJIB BOUSSETILA AND FAOUZIA REBBANI
}

\begin{abstract}
The goal of this paper is to present some extensions of the method of quasi-reversibility applied to an ill-posed Cauchy problem associated with an unbounded linear operator in a Hilbert space. The key point to our proof is the use of a new perturbation to construct a family of regularizing operators for the considered problem. We show the convergence of this method, and we estimate the convergence rate under a priori regularity assumptions on the problem data.
\end{abstract}

2000 Mathematics Subject Classification: 35K90, 47D06; 47A52, $35 \mathrm{R} 25$.

Key words and phrases: Ill-posed Cauchy problem, quasi-reversibility method, regularizing family.

\section{Introduction And Motivation}

Throughout this paper $H$ denotes a complex Hilbert space endowed with the inner product $(.,$.$) and the norm \|\|,. \mathcal{L}(H)$ stands for the Banach algebra of bounded linear operators on $H$.

Let $A$ be a linear unbounded operator with dense domain $\mathcal{D}(A)$. Assume that $A$ is self-adjoint, positive definite in $H$. We consider the abstract final value problem

$$
u^{\prime}(t)+A u(t)=0, \quad 0<t<T, \quad u(T)=f,
$$

where $f$ is a given function in $H$.

Such problems are not well-posed in the Hadamard sense [15]; the solution (if it exists) does not depend continuously on the data.

Physically, problems of this nature arise in different contexts. Beyond their interest in connection with standard diffusion problems (then $A$ is usually the Laplace operator $-\Delta$ ), they also appear, for instance, in some deconvolution problems, such as deblurring processes $[6](A$ is often a fractional power of $-\Delta)$, in hydrology $[16,36]$ and also in many other practical applications of mathematical physics and engineering sciences.

Since the semigroup $S(t)=e^{-t A}$ is not time-inverted, to obtain a well-posed problem, we would like to find an operator $S_{\alpha}(t), \alpha>0$, "close to" $S(t)$ in some sense for which the final value problem $(F V P)$ is well-posed.

In the mathematical literature various methods have been proposed for solving backward Cauchy problems. We can notably mention the method of quasisolution of Tikhonov [37], the method of quasi-reversibility of Lattès and Lions 
[23], the method of logarithmic convexity [1, 7, 21, 25, 30], the iterative procedures of Kozlov and Maz'ya [5, 22], the quasi boundary value method [8, 11, 20], the $C$-regularized semigroups theory [3, 26, 27, 33].

In the method of quasi-reversibility, the main idea consists in replacing $A$ in $(F V P)$ by $A_{\alpha}=g_{\alpha}(A)$. In the original method [23] Lattès and Lions proposed $g_{\alpha}(A)=A-\alpha A^{2}$, and in the Gajewski and Zaccharias quasi-reversibility method $[12,14,19,34], g_{\alpha}(A)=A(I+\alpha A)^{-1}$, to obtain a well-posed problem in the backward direction. Then, using the information from the solution of the perturbed problem, another well-posed problem is constructed, and its solution sometimes can be taken to be an approximate solution of the original ill-posed problem $(F V P)$.

Difficulties may arise when using the method of quasi-reversibility discussed above. an essential difficulty is that the operator coefficient is replaced by an operator of second order, which makes the numerical implementation rather difficult; in addition, the error $(e(\alpha))$ introduced by a small changes in the final value $f$ is of order $e^{\frac{1}{\alpha}}$. For these reasons, we propose a modified quasireversibility based on the perturbation

$$
A_{\alpha}=g_{\alpha}(A)=-\frac{1}{p T} \log \left(\alpha+e^{-p T A}\right), \quad \alpha>0, \quad p \geq 1 .
$$

An advantage of this new perturbation lies in the fact that it is bounded ( $A_{\alpha} \in$ $\mathcal{L}(H)$ ), which gives the well-posedness in the forward and backward direction for the perturbed problem, while another advantage is that this perturbation provides the best possible approximate solution, while the amplification factor of the error resulting from the approximated problem is better as compared with other results. We note that our approach generalizes many results obtained by other methods.

\section{Preliminaries and Basic Results}

In this section, we give the notation and functional is needed in the sequel.

If $B \in \mathcal{L}(H)$, we denote by $\mathcal{N}(B)$ the kernel of $B$ and by $\mathcal{R}(B)$ the range of $B$. We denote by $\left\{E_{\lambda}, \lambda \geq \gamma>0\right\}$ the spectral resolution of the identity associated to $A$, and by $S(t)=e^{-t A}=\int_{\gamma}^{\infty} e^{-t \lambda} d E_{\lambda} \in \mathcal{L}(H), t \geq 0$, the $C_{0^{-}}$ semigroup generated by $-A$. Some basic properties of $S(t)$ are listed in the following theorem:

Theorem 2.1 (see [32], Ch. 2, Theorem 6.13, p. 74). For the family of operators $S(t)$, the following properties are valid:

(1) $\|S(t)\| \leq 1, \quad \forall t \geq 0$;

(2) the function $t \longmapsto S(t), t>0$, is analytic;

(3) for every real $r \geq 0$ and $t>0$, the operator $S(t) \in \mathcal{L}\left(H, \mathcal{D}\left(A^{r}\right)\right)$;

(4) for every integer $k \geq 0$ and $t>0,\left\|S^{(k)}(t)\right\|=\left\|A^{k} S(t)\right\| \leq c(k) t^{-k}$;

(5) for every $x \in \mathcal{D}\left(A^{r}\right), r \geq 0$ we have $S(t) A^{r} x=A^{r} S(t) x$. 
Theorem 2.2. For every $t>0$, the operator $S(t)$ is self-adjoint and one-toone with dense range $\left(S(t)=S(t)^{*}, \mathcal{N}(S(t))=\{0\}\right.$ and $\left.\overline{\mathcal{R}(S(t))}=H\right)$.

Proof. $A$ is self-adjoint and since $S(t)^{*}=\left(e^{-t A}\right)^{*}=e^{-t A^{*}}=e^{-t A}$, we have $S(t)^{*}=S(t)$. Let $h \in \mathcal{N}\left(S\left(t_{0}\right)\right), t_{0}>0$, then $S\left(t_{0}\right) h=0$, which implies that $S(t) S\left(t_{0}\right) h=S\left(t+t_{0}\right) h=0, t \geq 0$. Using analyticity, we obtain that $S(t) h=0, t \geq 0$. Strong continuity at 0 now gives $h=0$. This shows that $\mathcal{N}\left(S\left(t_{0}\right)\right)=\{0\}$. By

$$
\overline{\mathcal{R}\left(S\left(t_{0}\right)\right)}=\mathcal{N}\left(S\left(t_{0}\right)\right)^{\perp}=\{0\}^{\perp}=H
$$

we conclude that $\mathcal{R}\left(S\left(t_{0}\right)\right)$ is dense in $H$.

For more details, we refer the reader to a general version of Theorem 2.2 in the case of analytic semigroups in Banach spaces (Lemma 2.2, [9]).

Remark 2.1. If we replace $A$ by $B=p A$ in Theorem 2.2, we obtain $\mathcal{N}(S(p t))=$ $\{0\}$ and $\overline{\mathcal{R}(S(p t))}=H, p>0, t>0$.

Remark 2.2 (Smoothing effect and irreversibility). By Theorems 2.1 and 2.2, we observe that the solution of the direct Cauchy problem:

$$
u^{\prime}(t)+A u(t)=0, \quad 0<t \leq T, \quad u(0)=u_{0},
$$

has the following smoothing effect: admitting the initial value $u(0)$ to belong only to $H$, for all $t>0$ we obtain

$$
\mathcal{R}(S(t)) \subset \mathcal{C}^{\infty}(A) \stackrel{\text { def }}{=} \cap_{n=1}^{\infty} \mathcal{D}\left(A^{n}\right)
$$

(a space more regular than $H$, see [13]). It follows that for the final value problem $(F V P)$ to have a solution, we should have $u(T) \in \mathcal{C}(A) \subseteq \mathcal{R}(S(T))$, where $\mathcal{C}(A)$ is an admissible class for which the $F V P$ is solvable. This shows that this problem is irreversible in the sense

$$
S(T-t): H \rightarrow \mathcal{R}(S(T-t)) \subset \mathcal{C}^{\infty}(A) \subsetneq H, \quad 0 \leq t<T,
$$

and $\mathcal{R}(S(T-t)) \neq \overline{\mathcal{R}(S(T-t))}$; in other words, $S(T-t)^{-1}=S(t-T) \notin \mathcal{L}(H)$.

For notational convenience and simplicity, we set

$$
\mathcal{C}_{\theta}(A)=\left\{\phi \in H:\|\phi\|_{\theta}^{2}=\int_{\gamma}^{+\infty} e^{2 T \theta \lambda} d\left\|E_{\lambda} \phi\right\|^{2}<+\infty\right\}, \quad \theta \geq 0 .
$$

Evidently,

$$
\mathcal{C}_{\theta_{2}}(A) \subseteq \mathcal{C}_{\theta_{1}}(A), \quad \theta_{2} \geq \theta_{1}
$$

We also need the following technical lemma.

Lemma 2.1. For $x \geq 0$ and $\tau \in[0,1]$, we have

$$
(1+x)^{\tau}-1 \leq \tau x(1+x)^{\tau}(1+\tau x)^{-1} .
$$

The proof is justified by a simple differential calculus (see [24], p. 101.)

Remark 2.3. The operational calculus for self-adjoint operators and Lemma 2.1 plays the key role in our analysis and calculations. 


\section{The Approximate Problem}

In this section, we give a constructive method based on the quasi-reversibility approach to construct a stable approximate solution to the ill-posed problem $(F V P)$.

\section{Description of the method:}

Step 1. Let $v_{\alpha}$ be a solution of the following perturbed problem

$$
v_{\alpha}^{\prime}(t)+A_{\alpha} v_{\alpha}(t)=0, \quad 0 \leq t<T, \quad v_{\alpha}(T)=f,
$$

where the operator $A$ is replaced by $A_{\alpha}$.

Step 2. We use the initial value

$$
v_{\alpha}(0)=\varphi_{\alpha}
$$

in the problem

$$
u_{\alpha}^{\prime}(t)+A u_{\alpha}(t)=0, \quad 0<t \leq T, \quad u_{\alpha}(0)=\varphi_{\alpha} . \quad(I V P)_{\alpha}
$$

Step 3. Finally, we show that

$$
\Phi_{\alpha}(f)=\left\|u_{\alpha}(T)-f\right\| \longrightarrow 0 \text { as } \alpha \longrightarrow 0 .
$$

\section{Analysis of the Method and Main Results}

4.1. Analysis of $A_{\alpha}$ and its consequences. We begin our study by giving some qualitative properties of $A_{\alpha}$.

For $0<\alpha \leq \alpha_{*}=1-e^{-\gamma T}, p \geq 1$, we define

$$
A_{\alpha}=g_{\alpha}(A):=-\frac{1}{p T} \log \left(\alpha+e^{-p T A}\right)=\int_{\gamma}^{\infty}-\frac{1}{p T} \log \left(\alpha+e^{-p T \lambda}\right) d E_{\lambda} .
$$

For more details concerning the logarithm of operators and its spectral properties see, e.g., [4, 17, 29].

Proposition 4.1. We have

(1) $A_{\alpha} \in \mathcal{L}(H)$ and $\left\|A_{\alpha}\right\| \leq \frac{1}{p T} \log \left(\frac{1}{\alpha}\right)$;

(2) $A_{\alpha}=A_{\alpha}^{*} \geq 0$ and $A_{\alpha} A^{\theta} v=A^{\theta} A_{\alpha} v, v \in \mathcal{D}\left(A^{\theta}\right), \theta \geq 0$;

(3) $\forall v \in \mathcal{D}(A), \lim _{\alpha \longrightarrow 0}\left\|A_{\alpha} v-A v\right\|=0$;

(4) $\forall v \in H, S_{\alpha}(t) v=e^{-t A_{\alpha}} v \longrightarrow S(t) v$ as $\alpha \longrightarrow 0$.

Proof. 1. The boundedness of the operator $A_{\alpha}$ follows immediately from the properties of $g_{\alpha}(\lambda), \lambda \geq \gamma$. Indeed, by the choice of $\alpha$ we have

$$
\alpha+e^{-p T \gamma} \leq \alpha+e^{-T \gamma} \leq 1
$$

which implies that

$$
\underline{g_{\alpha}}=g_{\alpha}(\gamma)=-\frac{1}{p T} \log \left(\alpha+e^{-p T \gamma}\right) \geq 0
$$


and

$$
\overline{g_{\alpha}}=\lim _{\lambda \longrightarrow+\infty} g_{\alpha}(\lambda)=-\frac{1}{p T} \log (\alpha)>0 .
$$

Observing that $g_{\alpha}^{\prime}(\lambda)>0$, we have $g_{\alpha} \nearrow$ and $\sup _{\lambda \geq \gamma} g_{\alpha}(\lambda)=\overline{g_{\alpha}}$. By using a spectral representation of $A_{\alpha}$ and the preceding remarks, we obtain the desired result.

2. Via the H-functional calculus (see [10]) and the self-adjointness of $A$ we establish (2).

3. Let $v \in \mathcal{D}(A)$, we have

$$
A_{\alpha} v=-\frac{1}{p T} \log \left(\alpha+e^{-p T A}\right) A^{-1} A v .
$$

Let us denote

$$
B_{\alpha}=-\frac{1}{p T} \log \left(\alpha+e^{-p T A}\right) A^{-1}=\int_{\gamma}^{+\infty} M_{\alpha}(\lambda) d E_{\lambda},
$$

where $M_{\alpha}(\lambda)=-\frac{1}{p T} \log \left(\alpha+e^{-p T \lambda}\right) \lambda^{-1}$. By the definition of $\alpha$, we observe that $M_{\alpha}(\lambda) \geq 0$ for all $\lambda \geq \gamma$ and

$$
M_{\alpha}(\lambda)=1-\frac{1}{p T} \log \left(1+\alpha e^{p T \lambda}\right) \lambda^{-1} \geq 0
$$

which implies that $M_{\alpha}(\lambda) \leq 1$ for all $\lambda \geq \gamma$. Consequently, the operator $B_{\alpha}$ is uniformly bounded, i.e., $\left\|B_{\alpha}\right\| \leq 1, \forall 0<\alpha \leq 1-e^{-\gamma T}$.

Let $v=e^{-p T A} h, h \in H$, we have

$$
\left\|\left(B_{\alpha}-I\right) v\right\|^{2}=\int_{\gamma}^{+\infty}\left(\frac{1}{p T} \log \left(1+\alpha e^{p T \lambda}\right) \lambda^{-1}\right)^{2} e^{-2 p T \lambda} d\left\|E_{\lambda} h\right\|^{2} .
$$

Since $\log (1+x) \leq x, x \geq 0$, then the quantity $(a)$ can be estimated as follows

$$
\left\|\left(B_{\alpha}-I\right) v\right\|^{2} \leq\left(\frac{\alpha}{p \gamma T}\right)^{2}\|h\|^{2} \longrightarrow 0, \text { as } \alpha \longrightarrow 0,
$$

from which it follows that $B_{\alpha} v \longrightarrow v$ in $H$ as $\alpha \longrightarrow 0, \forall v \in \mathcal{R}(S(p T))$. But $\mathcal{R}(S(p T))$ is dense in $H$ and $B_{\alpha}$ is uniformly bounded on $H$, hence, by continuity,

$$
\forall v \in H, \quad B_{\alpha} v \longrightarrow v, \text { as } \alpha \longrightarrow 0 .
$$

In particular, for $v \in \mathcal{D}(A)$ we obtain

$$
A_{\alpha} v=B_{\alpha} A v \longrightarrow A v, \text { as } \alpha \longrightarrow 0 .
$$

4. Since $A_{\alpha} \in \mathcal{L}(H)$, we can define

$$
S_{\alpha}(t)=e^{-t A_{\alpha}}=\left(\alpha+e^{-p T A}\right)^{\frac{t}{p T}}=\sum_{n=0}^{+\infty} \frac{(-t)^{n}}{n !} A_{\alpha}^{n}, \quad t \in \mathbb{R} .
$$


It is obvious to see that $\left\|S_{\alpha}(t)\right\| \leq 1, t \geq 0$. Thus $S_{\alpha}(t), t \geq 0$ is a strongly continuous semigroup of contraction on $H$. Also, $\frac{d}{d t} S_{\alpha}(t)=-A_{\alpha}(t) S_{\alpha}(t)$ and

$$
\begin{aligned}
S_{\alpha}(t)-S_{\beta}(t) & =\int_{0}^{t} \frac{d}{d \tau}\left(S_{\beta}(t-\tau) S_{\alpha}(\tau)\right) d \tau \\
& =\int_{0}^{t}\left(S_{\beta}(t-\tau) S_{\alpha}(\tau)\left(A_{\beta}-A_{\alpha}\right)\right) d \tau .
\end{aligned}
$$

Then $\forall t \geq 0,0<\alpha, \beta \leq 1-e^{-\gamma T}, h \in \mathcal{D}(A)$, we have

$$
\left\|S_{\alpha}(t) h-S_{\beta}(t) h\right\| \leq t\left\|A_{\beta} h-A_{\alpha} h\right\|
$$

which shows that $\left\{S_{\alpha}(t) h\right\}$ is a Cauchy sequence in $H$, uniformly in $t \in[0, T]$ (by virtue of (3) in Proposition 4.1).

To complete the proof of (4), observe that $S_{\alpha}(t)$ is a contraction and $\mathcal{D}(A)$ is dense in $H$, so the limit

$$
S_{\alpha}(t) h \longrightarrow \widetilde{S}(t) h \text { as } \alpha \longrightarrow 0, \quad t \geq 0,
$$

extends to all $h \in H$, and holds uniformly in $t \in[0, T]$. It is clear that $\widetilde{S}(t) \in$ $\mathcal{L}(H)$ is a strongly continuous semigroup of contraction on $H$.

Let $h \in \mathcal{D}(A), t>0$, then

$$
\begin{aligned}
\left\|S(t) h-S_{\alpha}(t) h\right\| & =\left\|\int_{0}^{t} \frac{d}{d \tau}\left(S(\tau) S_{\alpha}(t-\tau) h\right) d \tau\right\| \\
& \leq \int_{0}^{t}\left\|S_{\alpha}(t-\tau)\left(A-A_{\alpha}\right) S(\tau) h\right\| d \tau \\
& \leq \int_{0}^{t}\left\|\left(A-A_{\alpha}\right) S(\tau) h\right\| d \tau .
\end{aligned}
$$

Now we use

$$
\left\|A_{\alpha} S(\tau) h\right\|=\left\|B_{\alpha} A S(\tau) h\right\| \leq\|S(\tau) A h\|
$$

to get

$$
\left\|\left(A_{\alpha}-A\right) S(\tau) h\right\|=\leq 2\|S(\tau) A h\|
$$

Since $\|S(\tau) A h\|$ is continuous, by the dominated convergence theorem we have

$$
\lim _{\alpha \longrightarrow 0}\left\|S(t) h-S_{\alpha}(t) h\right\| \leq \int_{0}^{t} \lim _{\alpha \longrightarrow 0}\left\|\left(A-A_{\alpha}\right) S(\tau) h\right\| d \tau=0,
$$

which implies that $S_{\alpha}(t) \longrightarrow S(t)=\widetilde{S}(t)$ on $\mathcal{D}(A)$ as $\alpha \longrightarrow 0$. According to the density of $\mathcal{D}(A)$ in $H$ we conclude that $S_{\alpha}(t) \longrightarrow S(t)=\widetilde{S}(t)$ on $H$ as $\alpha \longrightarrow 0$. 
We note here that by a direct calculation with the help of Lemma 2.1 we can show

$$
\forall h \in H, \quad S_{\alpha}(t) h \longrightarrow S(t) h \text { as } \alpha \longrightarrow 0 .
$$

Indeed, let $v=e^{-p t A} h, h \in H$, we have

$$
\left\|S_{\alpha}(t) v-S(t) v\right\|^{2}=\int_{\gamma}^{+\infty}\left(\left(\alpha+e^{-p T \lambda}\right)^{\frac{t}{p T}}-e^{-t \lambda}\right)^{2} e^{-2 p T \lambda} d\left\|E_{\lambda} h\right\|^{2} .
$$

By virtue of Lemma 2.1 and $\alpha+e^{-p T \lambda} \leq 1$, the function

$$
M_{\alpha}(\lambda)=\left(\alpha+e^{-p T \lambda}\right)^{\frac{t}{p T}}-e^{-t \lambda}=e^{-t \lambda}\left(\left(1+\alpha e^{p T \lambda}\right)^{\frac{t}{p T}}-1\right)
$$

can be estimated as follows:

$$
M_{\alpha}(\lambda) \leq \frac{\frac{t}{p T} \alpha e^{p T \lambda}\left(\alpha+e^{-p T \lambda}\right)^{\frac{t}{p T}}}{\left(1+\frac{t}{p T} \alpha e^{p T \lambda}\right)} \leq \frac{\alpha}{p} e^{p T \lambda} .
$$

From this we derive

$\left\|S_{\alpha}(t) v-S(t) v\right\|^{2}=\int_{\gamma}^{+\infty} M_{\alpha}(\lambda)^{2} e^{-2 p T \lambda} d\left\|E_{\lambda} h\right\|^{2} \leq\left(\frac{\alpha}{p}\right)^{2}\|h\|^{2} \longrightarrow 0$ as $\alpha \longrightarrow 0$.

According to the density of $\mathcal{R}(S(p T))$ in $H$ and $\left\|S_{\alpha}(t)\right\| \leq 1, t \geq 0$, we conclude that

$$
\forall h \in H, \quad S_{\alpha}(t) h \longrightarrow S(t) h \text { as } \alpha \longrightarrow 0 .
$$

The proof of Proposition 4.1 is complete.

4.2. Convergence results. Now we are ready to state and prove the main results of this paper.

It is useful to know exactly the admissible set for which the problem $(F V P)$ has a solution. The following lemma answers this question.

Lemma 4.1 (see [8], Lemma 1). The problem (FVP) has a solution if and only if $f \in \mathcal{C}_{1}(A)$, and its unique solution is represented by

$$
u(t)=e^{(T-t) A} f .
$$

By using the semi-groups theory and the properties of $S_{\alpha}(t)$ we have the following theorems.

Theorem 4.1. For all $f \in H$, the function

$$
v_{\alpha}=e^{(T-t) A_{\alpha}} f=\left(\alpha+e^{-p T A}\right)^{-\frac{(T-t)}{p T}} f
$$

is a unique solution of the problem $(F V P)_{\alpha}$ and it depends continuously on $f$. 
To show the continuous dependence of $v_{\alpha}$ on $f$, we note that

$$
\begin{aligned}
\left\|v_{\alpha}(t)\right\| & =\left\|\left(\alpha+e^{-p T A}\right)^{-\frac{(T-t)}{p T}} f\right\| \\
& \leq\left(\frac{1}{\alpha}\right)^{\frac{T-t}{p T}}\|f\| \leq\left(\frac{1}{\alpha}\right)^{\frac{1}{p}}\|f\|=e(\alpha)\|f\| .
\end{aligned}
$$

From (2) we construct

$$
\varphi_{\alpha}=v_{\alpha}(0)=\left(\alpha+e^{-p T A}\right)^{-\frac{1}{p}} f .
$$

Theorem 4.2. The problem $(I V P)_{\alpha}$ is well-posed, and its solution is represented by

$$
u_{\alpha}(t)=S(t) \varphi_{\alpha}=e^{-t A}\left(\alpha+e^{-p T A}\right)^{-\frac{1}{p}} f .
$$

Theorem 4.3. For all $f \in H\left\|u_{\alpha}(T)-f\right\| \longrightarrow 0$ as $\alpha \longrightarrow 0$.

Proof. We compute

$$
\left\|u_{\alpha}(T)-f\right\|^{2}=\int_{\gamma}^{+\infty} H_{\alpha}(\lambda)^{2} d\left\|E_{\lambda} f\right\|^{2}
$$

where

$$
H_{\alpha}(\lambda)=\frac{\left(\left(\alpha+e^{-p T \lambda}\right)^{\frac{1}{p}}-e^{-T \lambda}\right)}{\left(\alpha+e^{-p T \lambda}\right)^{\frac{1}{p}}}=\frac{e^{-T \lambda}\left(\left(\alpha e^{p T \lambda}+1\right)^{\frac{1}{p}}-1\right)}{\left(\alpha+e^{-p T \lambda}\right)^{\frac{1}{p}}} .
$$

If we put $x=\alpha e^{p T \lambda}, \tau=\frac{1}{p}$, then by virtue of Lemma 2.1, the function $H_{\alpha}(\lambda)$ can be estimated as

$$
H_{\alpha}(\lambda) \leq \frac{\alpha}{\alpha+p e^{-p T \lambda}}
$$

From (6) it follows

$$
\left\|u_{\alpha}(T)-f\right\|^{2} \leq \int_{\gamma}^{+\infty}\left\{\frac{\alpha}{\alpha+p e^{-p T \lambda}}\right\}^{2} d\left\|E_{\lambda} f\right\|^{2} .
$$

Fix $\varepsilon>0$, and choose $N$ so that $\int_{N}^{+\infty} d\left\|E_{\lambda} f\right\|^{2}<\frac{\varepsilon}{2}$. Thus

$$
\begin{aligned}
\left\|u_{\alpha}(T)-f\right\|^{2} \leq & \int_{\gamma}^{N}\left\{\frac{\alpha}{\alpha+p e^{-p T \lambda}}\right\}^{2} d\left\|E_{\lambda} f\right\|^{2} \\
& +\int_{N}^{+\infty}\left\{\frac{\alpha}{\alpha+p e^{-p T \lambda}}\right\}^{2} d\left\|E_{\lambda} f\right\|^{2},
\end{aligned}
$$


which gives

$$
\mid u_{\alpha}(T)-f\left\|^{2} \leq\left(\frac{\alpha}{p}\right)^{2} e^{2 p T N}\right\| f \|^{2}+\frac{\varepsilon}{2} .
$$

So, by taking $\alpha$ such that $\left(\frac{\alpha}{p}\right)^{2} e^{2 p T N}\|f\|^{2}<\frac{\varepsilon}{2}$, we end the proof.

Remark 4.1. Here we do not have a convergence rate.

Theorem 4.4. If $f \in \mathcal{C}_{\theta}(A), p \geq 1,0<\theta<1$, then $\left\|u_{\alpha}(T)-f\right\|$ converges to zero with order $\alpha^{\frac{\theta}{p}}$.

Proof. We compute

$$
\left\|u_{\alpha}(T)-f\right\|^{2}=\int_{\gamma}^{+\infty}\left\{\frac{H_{\alpha}(\lambda)}{e^{\theta T \lambda}}\right\}^{2} e^{2 \theta T \lambda} d\left\|E_{\lambda} f\right\|^{2} \leq \int_{\gamma}^{+\infty} G_{\alpha}(\lambda)^{2} e^{2 \theta T \lambda} d\left\|E_{\lambda} f\right\|^{2},
$$

where

$$
G_{\alpha}(\lambda)=\frac{\alpha}{\left(\alpha+p e^{-p T \lambda}\right) e^{\theta T \lambda}}>0 .
$$

Differentiation with respect to $\lambda$ yields

$$
G_{\alpha}^{\prime}(\lambda)=\frac{\alpha T e^{\theta T \lambda}\left(p(p-\theta) e^{-p T \lambda}-\theta \alpha\right)}{\left(\left(\alpha+p e^{-p T \lambda}\right) e^{\theta T \lambda}\right)^{2}}
$$

Thus $G_{\alpha}^{\prime}(\lambda)=0$ if $\lambda=\lambda^{*}=\frac{1}{p T} \log \left(\frac{p(p-\theta)}{\theta \alpha}\right)$. Since $G_{\alpha}^{\prime}(\lambda)>0$ if $\lambda<\lambda^{*}$, $G_{\alpha}^{\prime}(\lambda)<0$ if $\lambda>\lambda^{*}$ and $\lim _{\lambda \rightarrow+\infty} G_{\alpha}(\lambda)=0$, we have that $\lambda^{*}$ is the critical value at which $G_{\alpha}$ achieves its maximum. Thus we have the inequality

$$
G_{\alpha}(\lambda) \leq G_{\alpha}\left(\lambda^{*}\right)=c(p, \theta) \alpha^{\frac{\theta}{p}}
$$

where $c(p, \theta)=\left(\frac{1}{p}\right)^{\frac{p+\theta}{p}}(p-\theta)^{\frac{p-\theta}{p}} \theta^{\frac{\theta}{p}} \leq 1$.

Combining (10) and (11), we arrive at

$$
\left\|u_{\alpha}(T)-f\right\| \leq c(p, \theta) \alpha^{\frac{\theta}{p}}\|f\|_{\theta} .
$$

Noting that in the case $1 \leq p \leq \theta$, we have the estimate

$$
\left\|u_{\alpha}(T)-f\right\| \leq \alpha\|f\|_{\theta}
$$

Theorem 4.5. For all $f \in H$, the problem $(F V P)$ has a solution $u$ if and only if the sequence $\varphi_{\alpha}=u_{\alpha}(0)$ converges in $H$. Furthermore, we then have $u_{\alpha}(t)$ converges to $u(t)$, as $\alpha$ tends to zero uniformly in $t$.

Proof. Assume that $\lim _{\alpha \rightarrow 0} \varphi_{\alpha}=\varphi_{0}$ exists. Let $w(t)=S(t) \varphi_{0}$. We compute

$$
\begin{aligned}
\left\|w(t)-u_{\alpha}(t)\right\| & =\left\|S(t) \varphi_{0}-S(t) \varphi_{\alpha}\right\|=\left\|S(t)\left(\varphi_{0}-\varphi_{\alpha}\right)\right\| \\
& \leq\left\|\varphi_{0}-\varphi_{\alpha}\right\| .
\end{aligned}
$$


This implies

$$
\sup _{0 \leq t \leq T}\left\|w(t)-u_{\alpha}(t)\right\| \leq\left\|\varphi_{0}-\varphi_{\alpha}\right\| \longrightarrow 0, \quad \text { as } \alpha \longrightarrow 0 .
$$

Since $\lim _{\alpha \longrightarrow 0} u_{\alpha}(T)=f$ and $\lim _{\alpha \longrightarrow 0} u_{\alpha}(T)=w(T)$, we infer that $w(T)=f$. Thus, $w(t)=S(t) \varphi_{0}$ solves the problem $(F V P)$ and satisfies the condition $w(T)=f$.

Now let us assume that $u(t)$ is a solution of $(F V P)$. From Lemma 4.1 we have $u(0)=S(-T) f \in H$, i.e., $\|u(0)\|^{2}=\|f\|_{1}^{2}=\int_{\gamma}^{+\infty} e^{2 T \lambda} d\left\|E_{\lambda} f\right\|^{2}<\infty$. Let $N>0$ and $\varepsilon>0$ such that $\int_{N}^{+\infty} e^{2 T \lambda} d\left\|E_{\lambda} f\right\|^{2}<\frac{\varepsilon}{2}$.

We compute

$$
\left\|u_{\alpha}(0)-u(0)\right\|^{2}=\int_{\gamma}^{+\infty} F_{\alpha}^{2}(\lambda) d\left\|E_{\lambda} f\right\|^{2}
$$

where

$$
F_{\alpha}(\lambda)=e^{T \lambda}-\left(\alpha+e^{-p T \lambda}\right)^{-\frac{1}{p}}
$$

By simple calculations with the help of Lemma 2.1, $F_{\alpha}(\lambda)$ can be estimated as

$$
F_{\alpha}(\lambda) \leq\left(\frac{\alpha e^{p T \lambda}}{p+\alpha e^{p T \lambda}}\right) e^{T \lambda}=K_{\alpha}(\lambda) e^{T \lambda} .
$$

Then

$$
\left\|u_{\alpha}(0)-u(0)\right\|^{2} \leq \int_{\gamma}^{+\infty} K_{\alpha}^{2}(\lambda) e^{2 T \lambda} d\left\|E_{\lambda} f\right\|^{2} \leq I_{1}+I_{2}
$$

where

$$
\begin{gathered}
I_{1}=\int_{\gamma}^{N} K_{\alpha}^{2}(\lambda) e^{2 T \lambda} d\left\|E_{\lambda} f\right\|^{2} \leq\left(\frac{\alpha}{p}\right)^{2} e^{2(p+1) T N}\|f\|^{2}, \\
I_{2}=\int_{N}^{+\infty} K_{\alpha}^{2}(\lambda) e^{2 T \lambda} d\left\|E_{\lambda} f\right\|^{2}<\frac{\varepsilon}{2}
\end{gathered}
$$

Now if we choose $\alpha$ such that $\left(\frac{\alpha}{p}\right)^{2} e^{2(p+1) T N}\|f\|^{2}<\frac{\varepsilon}{2}$, then we have

$$
\left\|u_{\alpha}(0)-u(0)\right\|^{2}<\varepsilon \text {. }
$$

This shows that

$$
u_{\alpha}(0) \longrightarrow u(0) \text { as } \alpha \longrightarrow 0 .
$$

Theorem 4.6. If $f \in \mathcal{C}_{1+\theta}(A), p \geq 1,0<\theta<1$, then $\left\|u_{\alpha}(0)-u(0)\right\|$ converges to zero with order $\alpha^{\frac{\theta}{p}}$. 
Proof. By similar calculations to those used in Theorems 4.4 and 4.5, we have

$$
\begin{aligned}
\left\|u_{\alpha}(0)-u(0)\right\|^{2} & \leq \int_{\gamma}^{+\infty} K_{\alpha}(\lambda)^{2} e^{-2 \theta T \lambda} e^{2(1+\theta) T \lambda} d\left\|E_{\lambda} f\right\|^{2} \\
& \leq \int_{\gamma}^{+\infty} G_{\alpha}(\lambda)^{2} e^{2(1+\theta) T \lambda} d\left\|E_{\lambda} f\right\|^{2} \\
& \leq\left(G_{\alpha}^{\infty}\right)^{2}\|f\|_{1+\theta}^{2},
\end{aligned}
$$

with $G_{\alpha}^{\infty}=\sup _{\lambda \geq \gamma} G_{\alpha}(\lambda) \leq c(p, \theta) \alpha^{\frac{\theta}{p}}$ (see estimate $(11)$ ).

From Theorems 4.5-4.6 we obtain

Corollary 4.1. If $f \in \mathcal{C}_{1+\theta}(A), \theta>0$, then $\left\|u_{\alpha}(t)-u(t)\right\|$ converges to zero with order $\alpha^{\frac{\theta}{p}}$ uniformly in $t$.

We end this paper by constructing a family of regularizing operators for $(F V P)$.

Definition 4.1. A family $\left\{R_{\alpha}(t), \alpha>0, t \in[0, T]\right\} \subset \mathcal{L}(H)$ is called a family of regularizing operators for the problem $(F V P)$ if for each solution $u(t)$, $0 \leq t \leq T$ of the $(F V P)$ with final element $f$, and for any $\delta>0$, there exists $\alpha(\delta)>0$, such that

$$
\begin{gathered}
\alpha(\delta) \longrightarrow 0, \quad \delta \longrightarrow 0 \\
\left\|R_{\alpha(\delta)}(t) f_{\delta}-u(t)\right\| \longrightarrow 0, \quad \delta \longrightarrow 0,
\end{gathered}
$$

for each $t \in[0, T]$ provided that $f_{\delta}$ satisfies $\left\|f_{\delta}-f\right\| \leq \delta$.

Define $R_{\alpha}(t)=e^{-t A}\left(\alpha+e^{-p T A}\right)^{-\frac{1}{p}}, t \geq 0, \alpha>0$; it is clear that $R_{\alpha}(t) \in$ $\mathcal{L}(H)$

In the following we will show that $R_{\alpha}(t)$ is a family of regularizing operators for the $(F V P)$.

Theorem 4.7. Assuming that $f \in \mathcal{C}_{1}(A)$, then $\left(\mathcal{R}_{2}\right)$ holds.

Proof. We have

$$
\begin{aligned}
H_{\alpha}(t) & =\left\|R_{\alpha}(t) f_{\delta}-u(t)\right\| \leq\left\|R_{\alpha}(t)\left(f_{\delta}-f\right)\right\|+\left\|R_{\alpha}(t) f-u(t)\right\| \\
& =\Delta_{1}(t)+\Delta_{2}(t),
\end{aligned}
$$

where

$$
\Delta_{1}(t)=\left\|R_{\alpha}(t)\left(f_{\delta}-f\right)\right\| \leq\left(\frac{1}{\alpha}\right)^{\frac{1}{p}} \delta
$$

and

$$
\Delta_{2}(t)=\left\|R_{\alpha}(t) f-u(t)\right\|
$$

Choose $\alpha=\sqrt{\delta}$, then $\alpha(\delta) \longrightarrow 0, \delta \longrightarrow 0$, and

$$
\Delta_{1}(t) \leq \delta^{\frac{2 p-1}{2 p}} \longrightarrow 0 \text { as } \delta \longrightarrow 0 .
$$


Now, by virtue of Theorem 4.5 we have

$$
\Delta_{2}(t)=\left\|u_{\alpha}(t)-u(t)\right\| \longrightarrow 0 \text { as } \delta \longrightarrow 0,
$$

uniformly in $t$. Combining (17) and (18) we obtain

$$
\sup _{0 \leq t \leq T}\left\|R_{\alpha}(t) f_{\delta}-f\right\| \longrightarrow 0 \text { as } \delta \longrightarrow 0 .
$$

This shows that $R_{\alpha}(t)$ is a family of regularizing operators for the $(F V P)$.

We proceed with the same technique used to establish the preceding results. We show that

with

$$
\Delta_{2}(t) \leq C(p, t, T) \alpha^{\frac{t}{p T}}\|f\|_{1}, \quad t>0
$$

$$
C(p, t, T)=p^{-\frac{p T+t}{p T}}(p T-t)^{\frac{p T-t}{p T}} t^{\frac{t}{p T}} T^{-1} \leq 1 .
$$

Example 4.1. We give an example to clarify our study. As an example we consider the following backward heat equation:

$$
\begin{aligned}
& \frac{\partial u(x, t)}{\partial t}-\frac{\partial^{2} u(x, t)}{\partial x^{2}}=0, \quad x \in(0, \pi), \quad t \in(0, T), \\
& u(0, t)=u(\pi, t)=0, \quad t \in(0, T), \\
& u(x, T)=\varphi(x), \quad x \in[0, \pi] .
\end{aligned}
$$

Here $u(x, t)$ represents the temperature at time $t$ at a point $x$ of a thin metal wire of length $\pi$.

The problem $(B H E)$ can be formulated in the abstract form as follows

$$
u^{\prime}(t)+A u(t)=0, \quad 0<t<T, \quad u(T)=\varphi,
$$

where the linear operator

$$
A: D(A) \subset H=L_{2}(0, \pi) \longrightarrow L_{2}(0, \pi)
$$

is defined by

with

$$
A:=-\frac{\partial^{2}}{\partial x^{2}}
$$

$$
\mathcal{D}(A):=H^{2}(0, \pi) \cap H_{0}^{1}(0, \pi) .
$$

It is easy to show that the operator $A$ is self-adjoint and positive with discrete spectrum $\left(\sigma(A)=\left\{\lambda_{n}\right\}_{n \geq 1}\right)$. We denote by $\left\{e_{n}\right\}_{n \geq 1}$ the orthonormal eigenbasis in $H$, associated to the eigenvalues $\left\{\lambda_{n}\right\}_{n \geq 1}$ such that:

$$
\begin{aligned}
& A e_{n}=\lambda_{n} e_{n}, \quad \lambda_{n}=n^{2}, \quad e_{n}(x)=\sqrt{\frac{2}{\pi}} \sin (n x), \quad n=1,2, \cdots \\
& \left(e_{n}, e_{m}\right)=\int_{0}^{\pi} e_{n}(x) e_{m}(x) d x=\frac{2}{\pi} \int_{0}^{\pi} \sin (n x) \sin (m x) d x= \begin{cases}1, & n=m, \\
0, & n \neq m .\end{cases} \\
& \forall f \in L_{2}(0, \pi), \quad f(x)=\sum_{n \geq 1} c_{n}(f) e_{n}(x)=\sum_{n \geq 1} \frac{2}{\pi} \int_{0}^{\pi} f(y) \sin (n y) d y \sin (n x) .
\end{aligned}
$$


By the functional calculus for the self-adjoint operator we have

$$
g(A)=\int_{\sigma(A)} g(\lambda) d E_{\lambda}=\sum_{n \geq 1} g\left(\lambda_{n}\right) P_{\lambda_{n}}, \quad P_{\lambda_{n}}(\xi)=c_{n}(\xi) e_{n}
$$

for each Borel-measurable function $g: \sigma(A) \longrightarrow \mathbb{C}$.

In particular, for $g_{\alpha}$ we obtain the perturbation

$$
A_{\alpha}=g_{\alpha}(A)=\sum_{n \geq 1}-\frac{1}{p T} \log \left(\alpha+e^{-p T \lambda_{n}}\right) P_{\lambda_{n}}
$$

and the associated semigroup

$$
S_{\alpha}(t)=e^{-t A_{\alpha}}=\sum_{n \geq 1}\left(\alpha+e^{-p T \lambda_{n}}\right)^{\frac{t}{p T}} P_{\lambda_{n}} .
$$

In this case, the approximate solution $u_{\alpha}(x, t)$ takes the form

$$
u_{\alpha}(x, t)=R_{\alpha}(t) f(x)=\frac{2}{\pi} \sum_{n \geq 1} e^{-t n^{2}}\left(\alpha+e^{-p T n^{2}}\right)^{\frac{-1}{p}} \int_{0}^{\pi} f(y) \sin (n y) d y \sin (n x) .
$$

\section{Concluding remarks and generalization.}

1. From (3) we observe that the error factor $e(\alpha)$ introduced by small changes in the final value $f$ is of order $\left(\frac{1}{\alpha}\right)^{\frac{1}{p}}, p \geq 1$.

2. In the case $p=1$ the representation of $u_{\alpha}($.$) coincides with that obtained$ in the method developed in [8], with the same error factor $e(\alpha)$ of order $\frac{1}{\alpha}$.

3. In [11] (resp. [14, 23]) the error factor $e(\alpha)$ is of order $\frac{1}{\alpha(1+\log (T / \alpha))}$ (resp. $\left.e^{\frac{1}{\alpha}}\right)$.

Observing that for $p>1,\left(\frac{1}{\alpha}\right)^{\frac{1}{p}}<e^{\frac{1}{\alpha}}, \quad\left(\frac{1}{\alpha}\right)^{\frac{1}{p}}<\frac{1}{\alpha(1+\log (T / \alpha))}$ for $\alpha>0$.

This shows that our approach has a nice regularizing effect and gives a better approximation as compared with the methods developed in $[8,11,14,23]$.

Let us consider

$$
\begin{aligned}
& u_{t}(t)+A u(t)=0, \quad 0<t<T, \quad u(0)=f, \\
& u_{t}(t)+A u(t)=0, \quad 0<t<T, \quad u(T)=f,
\end{aligned}
$$

where $A$ is a self-adjoint, linear unbounded operator in $H$ and changes the sign with $0 \in \rho(A)\left(A^{-1}\right.$ exists and $\left.A^{-1} \in \mathcal{L}(H)\right)$.

The spectral theory of self-adjoint operators enables us to write

$$
h=\int_{\mathbb{R}} d E_{\lambda} h=\int_{\mathbb{R}_{-}} d E_{\lambda} h+\int_{\mathbb{R}_{+}} d E_{\lambda} h=h_{-}+h_{+}, \quad h \in H,
$$

i.e., the Hilbert space $H$ decomposes into the direct sum $H=H_{-} \oplus H_{+}$, and

$$
A=\int_{\mathbb{R}} \lambda d E_{\lambda}=\int_{\mathbb{R}_{-}} \lambda d E_{\lambda}+\int_{\mathbb{R}_{+}} \lambda d E_{\lambda}=A_{-}+A_{+} \cdot
$$


It is well known that the problem $(C P)_{1}$ (resp. $\left.(C P)_{2}\right)$ is incorrectly posed in the sense of Hadamard. In order to regularize this problem, we propose the following family of operators:

$$
\begin{aligned}
R_{\alpha}(t)= & e^{(T-t) A_{-}}\left(\alpha e^{p T A_{-}}+(1-\alpha)\right)^{-\frac{1}{p}} \\
& +e^{-t A_{+}}\left(\alpha+(1-\alpha) e^{-p T A_{+}}\right)^{-\frac{1}{p}} \\
= & R_{\alpha}^{-}(t)+R_{\alpha}^{+}(t), \quad 0<\alpha<1, \quad p \geq 1 .
\end{aligned}
$$

We conclude our study with the following lemma (fundamental lemma).

Lemma 4.2. If $f=f_{-}+f_{+} \in H$, then $(C P)_{1}$ (resp. $\left.(C P)_{2}\right)$ has a solution if and only if

$$
\left.\int_{\mathbb{R}_{-}} e^{-2 T \lambda} d\left\|E_{\lambda} f_{-}\right\|^{2}<+\infty \quad \text { (resp. } \int_{\mathbb{R}_{+}} e^{2 T \lambda} d\left\|E_{\lambda} f_{+}\right\|^{2}<+\infty\right) .
$$

Proof. If $\int_{\mathbb{R}_{-}} e^{-2 T \lambda} d\left\|E_{\lambda} f_{-}\right\|^{2}<+\infty$, we define $u(t)=e^{-t A_{-}} f_{-}+e^{-t A_{+}} f_{+}$. It is not difficult to verify that $u(t)$ satisfies $(C P)_{1}$. Conversely, let $u(t)$ be a solution to $(C P)_{1}$. Then $u(T)=h=h_{-}+h_{+}=e^{-T A_{-}} f_{-}+e^{-T A_{+}} f_{+} \in H$. This implies that $h_{-}=e^{-T A_{-}} f_{-} \in H$, i.e., $\left\|h_{-}\right\|^{2}=\int_{\mathbb{R}_{-}} e^{-2 T \lambda} d\left\|E_{\lambda} f_{-}\right\|^{2}<+\infty$. In a similar way we show the second part of the lemma .

We define $u_{\alpha}(t)=R_{\alpha}(t) f$. With the same methodology used to establish the results of convergence in the case where $A$ is positive, we show that

$$
\begin{aligned}
& u_{\alpha}(T) \longrightarrow f \text { as } \alpha \longrightarrow 0, \\
& u_{\alpha}(0) \longrightarrow f \text { as } \alpha \longrightarrow 1,
\end{aligned}
$$

and $R_{\alpha}(t)$ is a family of regularizing operators for $(C P)_{1}\left(\right.$ resp. $\left.(C P)_{2}\right)$.

\section{ACKNOWLEDGMENTS}

The authors thank the anonymous referees for a thorough reading and corrections proposed, which improved the quality of the paper.

\section{REFERENCES}

1. S. Agmon and L. Nirenberg, Properties of solutions of ordinary differential equations in Banach space. Comm. Pure Appl. Math. 16(1963), 121-239.

2. K. A. Ames and L. E. Payne, Continuous dependence on modeling for some well-posed perturbations of the backward heat equation. J. Inequal. Appl. 3(1999), No. 1, 51-64.

3. K. A. Ames and R. J. Hughes, Structural stability for ill-posed problems in Banach space. Semigroup Forum 70(2005), No. 1, 127-145.

4. K. N. BoyAdzhiEv, Logarithms and imaginary powers of operators on Hilbert spaces. Collect. Math. 45(1994), No. 3, 287-300.

5. J. BAumeister and A. Leitão, On iterative methods for solving ill-posed problems modeled by partial differential equations. J. Inverse Ill-Posed Probl. 9(2001), No. 1, 1329 . 
6. A. S. Carasso, J. G. Sanderson, and J. M. Hyman, Digital removal of random media image degradations by solving the diffusion equation backwards in time. SIAM J. Numer. Anal. 15(1978), No. 2, 344-367.

7. A. S. Carasso, Logarithmic convexity and the "slow evolution" constraint in ill-posed initial value problems. SIAM J. Math. Anal. 30(1999), No. 3, 479-496 (electronic).

8. G. W. Clark and S. F. Oppenheimer, Quasireversibility methods for non-well-posed problems. Electron. J. Differential Equations 1994, No. 08, approx. 9 pp. (electronic).

9. I. Cioranescu and V. Keyantuo, Entire regularizations of strongly continuous groups and products of analytic generators. Proc. Amer. Math. Soc. 128(2000), No. 12, 35873593.

10. N. Dunford and J. T. Schwartz, Linear operators. Part I. General theory. Wiley Classics Library. A Wiley-Interscience Publication. John Wiley \& Sons, Inc., New York, 1988.

11. M. Denche and K. Bessila, A modified quasi-boundary value method for ill-posed problems. J. Math. Anal. Appl. 301(2005), No. 2, 419-426.

12. R. E. EwIng, The approximation of certain parabolic equations backward in time by Sobolev equations. SIAM J. Math. Anal. 6(1975), 283-294.

13. V. I. Gorbachuk, Spaces of infinitely differentiable vectors of a nonnegative selfadjoint operator. (Russian) Ukrain. Mat. Zh. 35(1983), No. 5, 617-621.

14. H. Gajewski and K. Zacharias, Zur Regularisierung einer Klasse nichtkorrekter Probleme bei Evolutionsgleichungen. J. Math. Anal. Appl. 38(1972), 784-789.

15. J. Hadamard, Lectures on Cauchy's problem in linear partial differential equations. Yale University Press, New Haven, 1923.

16. A. Hasanov and J. L. Mueller, A numerical method for backward parabolic problems with non-selfadjoint elliptic operators. Appl. Numer. Math. 37(2001), No. 1-2, 55-78.

17. M. HAase, Spectral properties of operator logarithms. Math. Z. 245(2003), No. 4, 761779.

18. Y. HuAng and Q. Zheng, Regularization for ill-posed Cauchy problems associated with generators of analytic semigroups. J. Differential Equations 203(2004), No. 1, 38-54.

19. Y. HuAng and Q. Zheng, Regularization for a class of ill-posed Cauchy problems. Proc. Amer. Math. Soc. 133(2005), No. 10, 3005-3012 (electronic).

20. V. K. Ivanov, I. V. Mel'nikova, and F. M. Filinkov, Operator-differential equations and ill-posed problems. (Russian) Fizmatlit "Nauka", Moscow, 1995.

21. F. John, Continuous dependence on data for solutions of partial differential equations with a presribed bound. Comm. Pure Appl. Math. 13(1960), 551-585.

22. V. A. Kozlov and V. G. MAZ'YA, Iterative procedures for solving ill-posed boundary value problems that preserve the differential equations. (Russian) Algebra $i$ Analiz 1(1989), No. 5, 144-170; English transl.: Leningrad Math. J. 1(1990), No. 5, 1207-1228

23. R. Lattè s and J.-L. Lions, Mé thode de quasi-ré versibilité et applications. Travaux et Recherches Mathématiques, No. 15. Dunod, Paris, 1967.

24. M. M. Lavrent'ev, V. G. Romanov, and S. P. Shishatskil, Ill-posed problems of mathematical physics and analysis. (Translated from the Russian) Translations of Mathematical Monographs, 64. American Mathematical Society, Providence, RI, 1986.

25. K. Miller, Stabilized quasi-reversibility and other nearly-best-possible methods for nonwell-posed problems. Symposium on Non-Well-Posed Problems and Logarithmic Convexity (Heriot-Watt Univ., Edinburgh, 1972), pp. 161-176. Lecture Notes in Math., Vol. 316, Springer, Berlin, 1973. 
26. I. V. MeL'Nikova, Q. Zheng, and J. Zheng, Regularization of weakly ill-posed Cauchy problems. J. Inverse Ill-Posed Probl. 10(2002), No. 5, 503-511.

27. I. V. Mel'nikova and S. V. Bochkareva, $C$-semigroups and the regularization of an ill-posed Cauchy problem. (Russian) Dokl. Akad. Nauk 329(1993), No. 3, 270-273; English transl.: Russian Acad. Sci. Dokl. Math. 47(1993), No. 2, 228-232

28. I. V. Mel'Nikova and A. I. Filinkov, Abstract Cauchy problems: three approaches. Chapman \& Hall/CRC Monographs and Surveys in Pure and Applied Mathematics, 120. Chapman \& Hall/CRC, Boca Raton, FL, 2001.

29. N. OKAZAWA, Logarithms and imaginary powers of closed linear operators. Integral Equations Operator Theory 38(2000), No. 4, 458-500.

30. L. E. PAYNE, Some general remarks on improperly posed problems for partial differential equations. Symposium on Non-Well-Posed Problems and Logarithmic Convexity (HeriotWatt Univ., Edinburgh, 1972), pp. 1-30. Lecture Notes in Math., Vol. 316, Springer, Berlin, 1973.

31. L. E. PAYNE, Improperly posed problems in partial differential equations. Regional Conference Series in Applied Mathematics, No. 22. Society for Industrial and Applied Mathematics, Philadelphia, Pa., 1975

32. A. PAZY, Semigroups of linear operators and applications to partial differential equations. Applied Mathematical Sciences, 44. Springer-Verlag, New York, 1983.

33. S. Piskarev, Estimates for the rate of convergence in the solution of ill-posed problems for evolution equations. (Russian) Izv. Akad. Nauk SSSR Ser. Mat. 51(1987), No. 3, 676-687; English transl.: Math. USSR-Izv. 30(1988), No. 3, 639-651.

34. R. E. Showalter, The final value problem for evolution equations. J. Math. Anal. Appl. $\mathbf{4 7}(1974), 563-572$.

35. R. E. Showalter, Cauchy problem for hyperparabolic partial differential equations. Trends in the theory and practice of nonlinear analysis (Arlington, Tex., 1984), 421-425, North-Holland Math. Stud., 110, North-Holland, Amsterdam, 1985.

36. T. H. SkagGs and Z. J. Kabala, Recovering the release history of a ground water contaminant plume: Method of quasi-reversibiliry. Water Resources Research 31(1995), No. 11, 2969-2973.

37. A. N. Tikhonov and V. Y. Arsenin, Solutions of ill-posed problems. (Translated from the Russian) Scripta Series in Mathematics. V. H. Winston \& Sons, Washington, D.C.: John Wiley \& Sons, New York-Toronto, Ont.-London, 1977.

(Received 14.11.2005; revised 10.09.2007)

Authors' address:

Applied Math Lab., University Badji Mokhtar-Annaba

P.O.Box. 12, Annaba 23000

Algeria

E-mails: n.boussetila@yahoo.fr

rebbani@wissal.dz 